\title{
NIVELES DE COMPRENSIÓN LECTORA Y SU RELACIÓN CON LA RESOLUCIÓN DE PROBLEMAS MATEMÁTICOS*
}

\author{
Aquino Palacios, Ingrid Maritza ${ }^{1}$ y Ríos Zea, Marta Celinda² \\ Facultad de Pedagogía y Humanidades de la Universidad Nacional del Centro de Perú \\ RESUMEN
}

A través de la presente investigación se ha propuesto comprender y valorar la importancia y la relación que tiene la lectura con el dominio de los códigos de las ciencias y las matemáticas. El problema detectado es que los alumnos en general presentan problemas de rendimiento académico en la solución de problemas matemáticos, por ello se planteó la siguiente interrogante ¿En qué medida influye el nivel de comprensión lectora en la capacidad de resolución de problemas matemáticos?. El objetivo general que motivó este trabajo fue: determinar el grado de influencia del nivel de comprensión lectora en la capacidad de resolución de problemas matemáticos. Entre los método utilizados se encuentra el método científico como general, y el descriptivo correlacional y el bibliográfico como métodos específicos. Se elaboraron instrumentos como fichas y prácticas de lectura y evaluaciones. La conclusión arribada es que se logró establecer es que existe una correlación positiva fuerte entre los niveles de comprensión lectora y la resolución de problemas matemáticos.

Palabras clave: niveles, comprensión lectora, problemas matemáticos, rendimiento, capacidad de resolución

\section{LEVELS OF READING COMPREMENSION UNDERSTANDING AND THEIR RELATION SHIP WITH THE RESOLUTION OF MATHEMICAL PROBLEMS}

\begin{abstract}
The present investigation has the propose of understanding and to value the importance the relationship reading has with learning the codes of science and mathemtics. The detected problem is that the students in general present problems of academic performance and the solution of $m A$ thematics problems. Fas this reason the following questions were considered: How the level of reading coMprehension has in the capacity of solving mathematical problems. The general influence of the level of reading comprehension in the capacity to solve mathematical problems. Among the methods used we found the scientific method as general and the conotional descriptive and bibliographical like specific methods. Instruments like cards practice sheets for reading and evaluation sneets were made. The conclusions that we achieved is that it was possible to establish a very strong positive relationship among the levels of reading understanding and the resolution of mathematical problems.
\end{abstract}

Key words: levels, reading comprebension, mathematical problemas, academic perfomance resolution capacity.

\section{INTRODUCCIÓN}

Ha sido necesario que en los países latinoamericanos se diera un largo proceso, forzosamente traumático, y jalonado, la mayoría de las veces, desde la evaluación mediante pruebas de comprensión lectora, para que apenas hoy se entendiera el verdadero sentido de leer.

Durante muchos años se creyó que el reconocimiento del alfabeto era suficiente para llamar a una persona lectora y considerada alfabetizada. Hoy sabemos que lector no es alguien que descifra las letras, y lee textos sencillos, aunque no tenga contacto con la cultura escrita. Lector, en sentido estricto, no es aquel o aquella que tiene prácticas lectoras superficiales, reducidas a lo estrictamente indispensable y a publicaciones periódicas con sobre información intrascendente.

\footnotetext{
* Este trabajo de investigación fue recibido el 20/01/2007 retornado para su revisión 20/06/2007 y aprobado para su publicación 10/12/2007.

1. Email: ingrid5555233@hotmail.com

2. Email. mrzea@hotmail.com
} 
Lector es una persona capaz de dialogar críticamente con diversos textos que circulan socialmente, y de tomar posturas frente a ellos, valorarlos e integrarlos en un mundo mental propio; lector es alguien habituado a leer libros, para quien los textos significan un desafío permanente, y que acepta ese reto y no se amedrenta ante los primeros obstáculos que le plantean, cualquiera que aquellos sea, incluyendo textos de origen audiovisual 0 virtual.

En este contexto donde hoy, leer no sólo es un derecho ciudadano de todos, sino un factor de sobrevivencia lingüística en un mundo donde miles de emisores lanzan intermitentemente mensajes esperando encontrar receptores o lectores que acepten el contenido de esos mensajes, proponemos el siguiente trabajo de investigación titulado "Niveles de comprensión lectora y su relación con la resolución de problemas matemáticos", con el único propósito de comprender la importancia y la relación que tiene la lectura en la resolución de problemas matemáticos, pues sabemos que el dominio de los códigos de las ciencias y las matemáticas es el dominio de la lectura. En el caso de las ciencias y las matemáticas leer implica saber leer sus anotaciones convencionales, saber combinarlas para producir diversas representaciones respecto a los problemas que se plantean: La resolución de ecuaciones es una práctica de abstracciones que tiene como fin dominar un universo, de las matemáticas, que ayuda a cualificar las formas del razonamiento matemático, o que, en la vida práctica, ayuda a cohesionar las formas lógicas del pensamiento.

Niveles de comprensión lectora y su relación con la resolución de problemas matemáticos", pretende dar respuesta a la siguiente interrogante ¿en qué medida influye el nivel de comprensión lectora en la capacidad de resolución de problemas matemáticos?.

El objetivo que motivó la culminación del presente trabajo de investigación fue: determinar el grado de influencia del nivel de comprensión lectora en la capacidad de resolución de problemas matemáticos.

Se desarrolló una investigación básica descriptiva correlacional.

La población estuvo constituida por los alumnos de IV ciclo de la Carrera de Educación Secundaria, especialidad de Matemática y Física de la Facultad de Pedagogía y Humanidades de la Universidad Nacional del Centro del Perú.

Las técnicas de investigación que se utilizaron en la investigación fueron: fichas de lectura y evaluaciones escritas.
El trabajo de investigación se consolidó en cuatro capítulos distribuidos de la siguiente manera:

El capítulo I, aborda el planteamiento del estudio, en el cual se considera la caracterización y formulación del problema, la justificación y la importancia así como los objetivos de investigación.

El capítulo II, comprende el marco teórico, en el cual se aborda los antecedentes, las bases teóricas y conceptuales para ubicar el tema dentro del contexto cognoscitivo.

En el capítulo III, se describen los aspectos metodológicos utilizados en la investigación: el tipo de investigación, el método, el diseño de la investigación, la identificación de la población y la muestra, además de las variables de estudio, y las técnicas, instrumentos, hipótesis y procedimientos de recolección de datos.

El capítulo IV del informe final, presenta el análisis y el tratamiento estadístico de los resultados y finalmente se presentan las conclusiones, sugerencias, bibliografía y los anexos.

\section{MATERIAL Y MÉTODOS}

\section{Tipo de investigación}

La presente investigación corresponde al tipo aplicada. Es aplicada porque su finalidad es obtener información pertinente sobre los niveles de la comprensión lectora en relación a la solución de problemas matemáticos, para actuar y modificar las consecuencias prácticas que de ella se deriven.

\section{Métodos y procedimiento de investigación}

La investigación tuvo como método general al científico, teniendo en cuenta sus procedimientos, planteamiento del problema, elaboración de las hipótesis y del marco teórico, recolección, análisis e interpretación de los datos, conclusiones y las sugerencias respectivas. Y como método específico se utilizó el descriptivo correlacional pues se describió los niveles de la comprensión lectora y se relacionó con la solución de problemas matemáticos.

\section{Diseño de investigación}

El diseño aplicado en la investigación fue de tipo correlacional, cuyo diagrama es el siguiente:

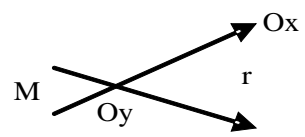


Donde:

M: Representa la muestra donde se ha realizado la investigación.

Ox: Representa la información o datos acerca de los niveles de la compresión lectora.

r: Representa la relación de los niveles de la compresión lectora y la resolución de problemas matemáticos que se obtuvo al aplicar el instrumento en la muestra respectiva.

Oy: Representa la información o datos obtenidos del rendimiento académico en la resolución de problemas matemáticos.

\section{Población y muestra}

- Población.- La población estuvo conformada por cinco secciones que hacían un total de 230 alumnos de la Carrera de Educación Secundaria, especialidad, Matemática y Física de la Facultad de Pedagogía y Humanidades.

- Muestra.- La muestra estuvo constituida por 32 alumnos del IV semestre y fue recogida mediante el muestreo no aleatorio según criterio de investigación.

\section{Técnicas e instrumento de investigación}

Durante el desarrollo de la investigación se aplicó la siguiente técnica de recopilación de datos:

Documental. Se consultó diversas fuentes bibliográficas relacionadas con la comprensión lectora y la resolución de problemas matemáticos.

\section{Material e instrumento de investigación}

Se utilizaron para el desarrollo de la investigación los siguientes instrumentos:

a) Prueba de comprensión lectora. Estuvo conformado por una lectura matemática acompañada por diversas preguntas que permitieron evaluar los niveles de la comprensión lectora. Se consideraron los siguientes niveles de la comprensión lectora: nivel literal o de traducción, nivel interpretativo o inferencial, y el nivel de extrapolación. El tipo de preguntas utilizadas en este instrumento fueron las interrogantes abiertas.

b) Prueba de resolución de problemas matemáticos: estuvo conformada por un conjunto de ejercicios que permitieron evaluar la relación de la comprensión lectora con la resolución de problemas matemáticos.

\section{Procedimiento de recolección de datos}

La recolección de los datos tuvo dos momentos:

Primero, de presentación y conocimiento del instrumento; se les habló de la importancia de la investigación y de dos problemas tan importantes que el Ministerio de Educación viene puntualizando: la comprensión lectora y el pensamiento lógico matemático, y que como investigadores queríamos encontrar específicamente dónde radica el problema en la resolución de problemas matemáticos.

Segundo, se les presentó el pre test y se les comunicó que contesten con sinceridad y se les explicó cómo deberían responder el cuestionario. El tiempo que duró la aplicación de este cuestionario fue de 30 minutos.

\section{Técnica de procesamiento y análisis de datos}

Los resultados y datos obtenidos con el instrumento de recolección de datos fueron organizados y tabulados de acuerdo a la variable de investigación, luego se analizaron con la escala de medición ordinal. Para el procesamiento estadístico se utilizó la estadística descriptiva.

Los datos recopilados fueron procesados bajo criterios científicos y metodológicos de la investigación, teniendo en cuenta:

\section{a) Estadística descriptiva}

Nos sirvió para describir y analizar los datos obtenidos una vez que culminó la aplicación de las pruebas de entrada y salida en los grupos:

\section{- Medidas de tendencia central}

Para el tratamiento de los datos obtenidos y la manera como estaban distribuidos de acuerdo a los valores centrales.

$$
\begin{aligned}
& \text { Medida aritmética } \overline{\mathrm{x}}=\sum \frac{\mathrm{X} 1 \mathrm{n} 1}{N} \\
& \text { - Medidas de dispersión } \\
& \text { - Varianza } \\
& S_{x}^{2}=\sum \frac{\left(x_{i}-\bar{x}\right)^{2} n_{i}}{N} \\
& \text { - Desviación típica } S_{x}=\sqrt{S_{x}^{2}} \\
& r=\frac{n \Sigma X Y-(\Sigma X)(\Sigma Y)}{\sqrt{\left[n \Sigma X^{2}-(\Sigma X)^{2}\right]\left[n \Sigma Y^{2}-(\Sigma Y)^{2}\right]}}
\end{aligned}
$$

\section{RESULTADOS}

\section{Análisis estadístico de los resultados}

Se hizo el tratamiento estadístico utilizando el paquete estadístico del MINITAB 14, así como la hoja de Cálculo 
EXCEL, se consideraron estadígrafos descriptivos tales como la media aritmética y la desviación típica, tanto en la prueba de comprensión lectora como en la prueba de resolución de problemas matemáticos.

La especialidad de Matemática y Física se caracteriza por tener más estudiantes varones que mujeres como podemos apreciar en la siguiente tabla:

Tabla 1.- Clasificación de alumnos por sexo

\begin{tabular}{lcc}
\hline SEXO & Alumnos & Porcentaje \\
\hline Masculino & 26 & $81 \%$ \\
Femenino & 6 & $19 \%$ \\
TOTAL & 32 & $100 \%$ \\
\hline
\end{tabular}

Fuente: Archivos de la Facultad

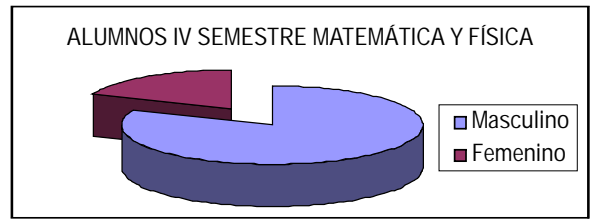

Gráfico 1.- Alumnos de Matemática y Física

El 81\% de los alumnos son de sexo masculino y sólo el 19\% son de sexo femenino.

A continuación, se presentan los resultados de las pruebas de comprensión lectora $(X)$ y de resolución de problemas matemáticos $(\mathrm{Y})$, con sus correspondientes estadísticos descriptivos y gráficos.

4.2 Análisis de los resultados de la comprensión lectora.

Tabla 2.- Niveles de la compresión lectora

\begin{tabular}{cc}
\hline $\mathrm{N}^{\circ}$ de alumnos & Nota \\
\hline 1 & 11,9 \\
1 & 10,1 \\
1 & 9,6 \\
1 & 8,6 \\
3 & 8,4 \\
1 & 7,9 \\
1 & 7,6 \\
2 & 6,7 \\
1 & 6,2 \\
1 & 6,0 \\
4 & 5,9 \\
2 & 5,4 \\
1 & 5,2 \\
2 & 5,1 \\
1 & 5,0 \\
1 & 4,4 \\
4 & 4,2 \\
1 & 3,4 \\
1 & 2,0 \\
1 & 1,7 \\
1 & 1,0 \\
\hline 32 & \\
\hline
\end{tabular}

Tabla 3.- Niveles de la compresión lectora

\begin{tabular}{cc}
\hline $\mathrm{N}^{\circ}$ de alumnos & Nota \\
\hline 1 & 12 \\
2 & 10 \\
1 & 09 \\
5 & 08 \\
2 & 07 \\
6 & 06 \\
6 & 05 \\
5 & 04 \\
1 & 03 \\
2 & 02 \\
1 & 01 \\
\hline 32 &
\end{tabular}

Tabla 4.- Niveles de la compresión lectora

\begin{tabular}{ccc}
\hline Alumnos & Número & $\%$ \\
\hline Aprobados & 1 & 3,1 \\
Desaprobados & 31 & 96,9 \\
Total & 32 & 100 \\
\hline
\end{tabular}

Tabla 5.- Respuestas incorrectas

\begin{tabular}{ccl}
\hline $\begin{array}{c}\text { No de } \\
\text { alumnos }\end{array}$ & Nota & \multicolumn{1}{c}{$\begin{array}{c}\text { Número de respuestas } \\
\text { incorrectas }\end{array}$} \\
\hline 1 & 12 & $7,8,9$ \\
1 & 10 & $3,5,8,9$ \\
1 & 10 & $2,3,8,9$ \\
1 & 09 & $2,3,8,9$ \\
3 & 08 & $3,7,8,9 ; 2,3,7,8,9 ; 3,7,8,9$ \\
1 & 08 & $2,3,5,8,9$ \\
1 & 08 & $2,3,4,5,7,8,9$ \\
2 & 07 & $3,5,6,7,8,9 ; 2,3,4,5,7,8,9$ \\
1 & 06 & $2,3,6,7,8,9$ \\
1 & 06 & $3,7,8,9$ \\
4 & 06 & $2,3,4,7,8,9 ; 2,3,4,6,8,9 ;$ \\
& & $2,3,5,7,8,9 ; 2,3,3,5,7,8,9$ \\
1 & 05 & $3,4,5,7,8,9$ \\
2 & 05 & $2,3,5,7,8,9 ; 3,5,6,7,8,9$ \\
2 & 05 & $2,3,4,5,8,9 ;: 2,3,4,5,8,9$ \\
1 & 05 & $3,6,7,8,9$ \\
1 & 04 & $2,3,4,5,6,7,8,9$ \\
4 & 04 & $2,3,5,6,7,8,9 ;$ \\
& & $2,3,4,6,7,8,9 ; 2,3,4,6,7,8$ \\
& & $9 ; 2,3,5,6,7,8,9$ \\
1 & 03 & $3,4,5,6,7,8,9$ \\
1 & 02 & $1,2,3,4,5,6,7,8,9$ \\
1 & 02 & $2,3,4,5,6,7,8,9$ \\
1 & 01 & $2,3,4,5,6,7,8,9$ \\
32 & &
\end{tabular}

De la tabla 2 podemos afirmar que el $98 \%$ de los alumnos están desaprobados. 
Tabla 6.- Respuestas incorrectas de acuerdo a los niveles de la lectura

\begin{tabular}{lccc}
\hline $\begin{array}{c}\text { Niveles de } \\
\text { compresión }\end{array}$ & $\begin{array}{c}\mathbf{N}^{\circ} \text { de } \\
\text { alumnos }\end{array}$ & $\begin{array}{c}\% \\
\text { alumno }\end{array}$ & $\begin{array}{c}\text { Respuestas } \\
\text { incorrectas } \\
\%\end{array}$ \\
\hline Nivel de & 14 & & 6 \\
traducción & 23 & & 7 \\
Total & 1 & & 1 \\
& 38 & 35.72 & \\
Nivel de & 23 & & 2 \\
Interpretación & 15 & & 3 \\
& 21 & & 4 \\
Total & 90 & 70.31 & 5 \\
& 32 & & 8 \\
Nivel de & 32 & & 9 \\
extrapolación & & & \\
Total & 64 & 100 & \\
TOTAL & 32 & & 100 \\
\hline
\end{tabular}

De la tabla 4 podemos deducir que el $100 \%$ de los alumnos no han contestado las preguntas números 8 y 9 , que corresponden al nivel de extrapolación. En segundo lugar con un $70 \%$, los alumnos no contestaron las preguntas 2 , 3,4 y 5 que corresponden al nivel de interpretación, mientras que el $35,72 \%$ de los alumnos no respondieron correctamente las preguntas de traducción 1,6 y 7 .

Estos resultados nos indican que la mayor dificultad que presentan los alumnos durante la comprensión lectora, en primer lugar, es el nivel de extrapolación, luego el de interpretación y en tercer lugar las preguntas de traducción.

\section{Estadística descriptiva:}

\section{COMPRENSIÓN LECTORA}

Donde podemos observar que en la prueba de comprensión lectora la media aritmética es 6 , la desviación típica es de 2,375, siendo los valores mínimos igual a 1 y máximo igual a 11,9 .

\begin{tabular}{ccccc}
\hline Variable & $\mathbf{N}$ & Promedio & \multicolumn{2}{c}{ Desv. Media } \\
\hline c1 & 32 & 6,044 & 0,420 & \\
\hline & & & & \\
\hline Variable & Desv. Est. & Mínimo & Mediana & Máximo \\
\hline c1 & 2,375 & 1,000 & 5,900 & 11,900 \\
\hline
\end{tabular}

Tabla 7.- Puntajes

\begin{tabular}{|c|c|c|}
\hline Orden & $X$ & $Y$ \\
\hline 1 & 6,7 & 12 \\
\hline 2 & 5,1 & 9 \\
\hline 3 & 8,4 & 8 \\
\hline 4 & 10,1 & 13 \\
\hline 5 & 5,0 & 5 \\
\hline 6 & 5,9 & 7 \\
\hline 7 & 6,0 & 12 \\
\hline 8 & 5,4 & 9 \\
\hline 9 & 9,6 & 11 \\
\hline 10 & 8,4 & 12 \\
\hline 11 & 2,0 & 3 \\
\hline 12 & 4,2 & 4 \\
\hline 13 & 6,2 & 12 \\
\hline 14 & 7,9 & 13 \\
\hline 15 & 4,2 & 8 \\
\hline 16 & 1,7 & 3 \\
\hline 17 & 4,4 & 8 \\
\hline 18 & 8,6 & 12 \\
\hline 19 & 8,4 & 12 \\
\hline 20 & 6,2 & 12 \\
\hline 21 & 1,0 & 4 \\
\hline 22 & 5,4 & 11 \\
\hline 23 & 4,2 & 6 \\
\hline 24 & 5,1 & 12 \\
\hline 25 & 4,2 & 7 \\
\hline 26 & 11,9 & 13 \\
\hline 27 & 5,9 & 9 \\
\hline 28 & 5,2 & 8 \\
\hline 29 & 7,6 & 10 \\
\hline 30 & 5,9 & 7 \\
\hline 31 & 5,9 & 9 \\
\hline 32 & 6,7 & 10 \\
\hline
\end{tabular}

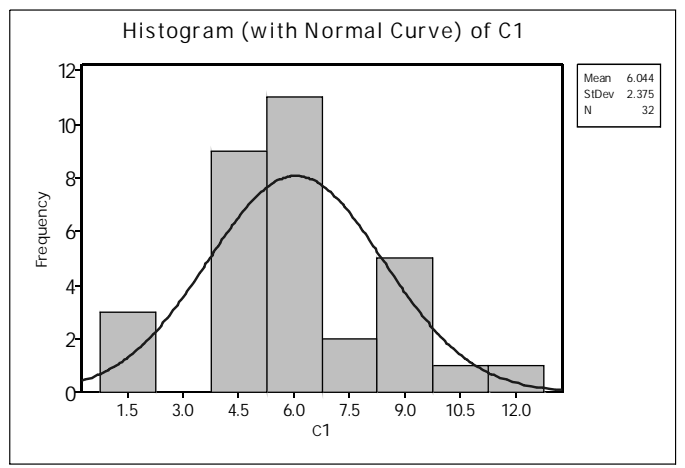

Gráfico 2.- Histograma de frecuencias de la prueba de comprensión lectora

Fuente: Archivo de investigadoras 


\section{Análisis de los resultados de la resolución de problemas}

Descriptive Statistics:

Resolución de problemas matemáticos

\begin{tabular}{cccc}
\hline Variable & $\mathbf{N}$ & Promedio & Desv. Media \\
\hline PM & 32 & 9,094 & 0.543 \\
\hline
\end{tabular}

\begin{tabular}{ccccc}
\hline Variable & Desv. Est. & Mínimo & Mediana & Máximo \\
\hline PM & 3,073 & 3,000 & 9,000 & 13,000 \\
\hline
\end{tabular}

En la prueba de problemas matemáticos se tiene una media aritmética de 9 con una desviación típica de 3,073, y los valores: mínimo de 3 y máximo de 13.

Acontinuación se presenta el gráfico correspondiente:

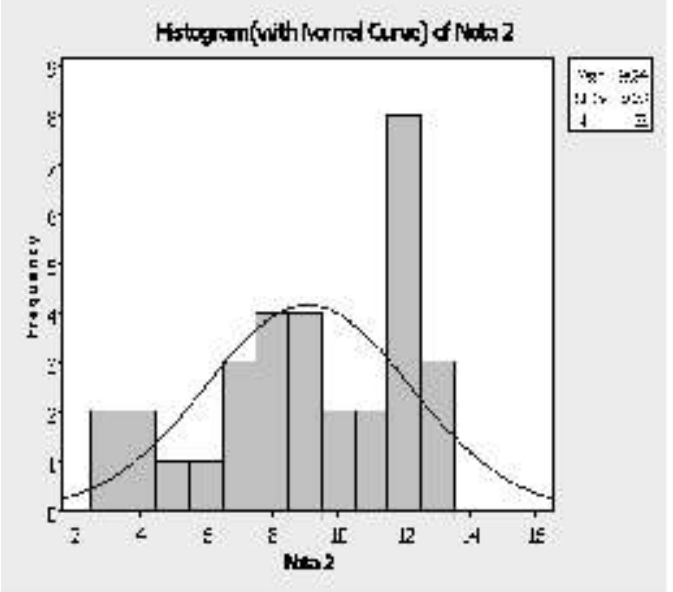

Gráfico 3.- Histograma de frecuencias de resolución de problemas matemáticos

Para verificar la existencia o no de relación se hizo uso del coeficiente de correlación $r$ de Pearson, teniendo como hipótesis nula:

Ho: No existe relación entre los niveles de comprensión lectora y la resolución de problemas matemáticos.

\section{$\mathrm{XrY}$}

Hi: Existe relación entre los niveles de comprensión lectoray la resolución de problemas matemáticos.

\section{$X r Y$}

Prueba estadística: coeficiente r de Pearson.

Correlación: Nota 1, Nota 2
Pearson correlation of Nota 1 and Nota $2=0,785$

$$
\mathrm{P} \text {-Valor }=0.000
$$

Toma de decisiones: si p valor $<0,05$ se acepta la hipótesis alterna y se rechaza la hipótesis nula, Por lo tanto se rechaza la hipótesis nula y se acepta la hipótesis alterna, obteniéndose un coeficiente de correlación de 0,785 , lo cuál indica una correlación positiva fuerte por lo tanto podemos concluir que si existe mucha relación entre los niveles de comprensión lectora y la resolución de problemas matemáticos.

\section{DISCUSIÓN}

Que el coeficiente de correlación sea positivo y fuerte, nos lleva a plantear que a mejores niveles de comprensión lectora, mejores posibilidades de resolver problemas matemáticos.

En cuanto a la investigación realizada por Véliz y Chipana, coincidimos en la conclusión donde señalan que una de las principales dificultades para resolver problemas es la incomprensión del enunciado e insuficiente familiarización con el mismo.

En cuanto a los alumnos de la muestra, encontramos que sus niveles de comprensión lectora no son los más adecuados influyendo ello en las dificultades que tienen para resolver los problemas matemáticos.

\section{CONCLUSIONES}

- Existe una correlación positiva fuerte con un coeficiente de correlación de Pearson $r=0,785$ entre los niveles de comprensión lectora y la resolución de problemas matemáticos, lo que indica que a mayores niveles de comprensión lectora, mayor facilidad en la resolución de problemas.

- La mayor dificultad que presentan los alumnos durante la comprensión lectora, es el nivel de extrapolación, luego el de interpretación y en tercer lugar las preguntas de traducción.

- La dificultad que presentan los alumnos en cuanto a la resolución de problemas, es en primer lugar, entender el enunciado y en segundo lugar traducir del lenguaje usual al lenguaje simbólico.

\section{LITERATURA CITADA}

Centro Pre Universitario. 2003. Razonamiento verbal. Universidad Nacional Agraria La Molina. Lima Perú.

De Guzmán, M. 2004. Tendencias innovadoras en educación matemática. Instituto Nacional de Ciencias de la Universidad de Zaragoza -España; p.33. 
Díaz B. F. Y Hernández, 1998. Estrategias Docentes para un Aprendizaje significativo. McGraw Hill. México.

Chi Y Glasser. 1983. Habilidades para la solución de problemas. Edit. Stemberg. New York1983.

Joven N. 2004. Editorial José Vicente Magisterio. № 7 Colombia.

Falk De Losada. 2000. María. La resolución de problemas como metodología. Artículo de la Revista, de Educación Matemática QUBO. Bachillerato peruano.

Flores O. R. 1994. Hacia una Pedagogía del conocimiento. Edit. Mc Graw Hill. Colombia.

Galindo S. L. y Roca M. E. 2003. Método de resolución de problemas en el aprendizaje de ángulos en el plano en alumnas del cuarto grado de educación secundaria en el Colegio Estatal de Mujeres "Nuestra Señora de Cocharcas"- Huancayo. Tesis. UNCP.

Gatti M. y Wiesse R. 2000. Técnicas de lectura y redacción. Universidad del Pacífico. Lima-Perú
Manual de Redacción. Editorial Universitaria. URP- Lima Perú.

Pérez G. H. 2001. Nuevas tendencias de la composición escrita. Edit. Aula abierta Magisterio. Colombia

Pinzás G. J. 2001. Leer pensando. Pontificia Universidad Católica del Perú. Fondo editorial. Lima-Perú.

Polya, G. 1969. Como plantear y resolver problemas. Edit. Trillas S.A. México, p. 215.

Sánchez L. D. y Solé, Isabel. 2004. Cómo leer mejor. IMLIL. Lima-Perú

Estrategias de Lectura. 2000. Edit. GRAÓ. España.

Uriarte M., Felipe.2005. Técnicas pata estudiar. Editorial San Marcos. Lima Perú.

Veliz T. R. y Chipana T. G., 1974. Un estudio sobre las dificultades para resolver problemas de matemática en los tres últimos años de educación secundaria común. Monografía. UNCP. 